\title{
Correction to: Stability of the Euler resting $N$-body relative equilibria
}

\author{
D. J. Scheeres ${ }^{1}$ (D)
}

Published online: 20 August 2018

(C) Springer Science+Business Media B.V., part of Springer Nature 2018

\section{Correction to: Celest Mech Dyn Astr (2018) 130:26 https://doi.org/10.1007/s10569-018-9819-7}

Due to an error in a supporting algorithm used in writing the paper there was an incorrect conclusion given which is corrected here. For the Euler resting $N$-body relative equilibrium the configuration can never be stable when $N \geq 22$, not $N \geq 6$ as reported in the original paper. This means that chains of up to 21 resting bodies can be stable if given the proper spin rate, and this places an ideal limit of 21:1 on the aspect ratio of a rubble pile body's shape.

The error occurred in the computation of the fission spin rate, which was stated correctly in the paper but which left off a factor of $\sqrt{2}$ in the supporting computational algorithm. When used to compute whether the Hessian was positive definite at $N=6$, the Hessian was found to not be positive definite, because the incorrect fission spin rate was too low and not beyond the stabilization spin rate.

As a result of this, the general conclusions are unchanged even though the specific limits are different. To correct the paper the following changes must be made.

In the Abstract section, the last three lines were published incorrectly. It should read as "This paper shows that the Euler Resting configuration can only be stable for up to 21 bodies and that for 22 or more bodies the configuration can never be stable. This places an ideal limit of $21: 1$ on the aspect ratio of a rubble pile body's shape.

In the original version, the article note was published incorrectly. The correct version should read as:

This article is part of the topical collection on Recent advances in the study of the dynamics of N-body problem. Guest Editors: Giovanni Federico Gronchi, Ugo Locatelli, Giuseppe Pucacco and Alessandra Celletti

The original article can be found online at https://doi.org/10.1007/s10569-018-9819-7.

D. J. Scheeres

scheeres@colorado.edu

1 Smead Department of Aerospace Engineering Sciences, The University of Colorado Boulder, 429 UCB, Boulder, CO, USA 
Under the section "1 Introduction", in paragraph 2, the last line should read as "That question is answered in the affirmative in this paper, with the answer being 21 ".

Under the section "1 Introduction", in paragraph 3, the second line should read as "In particular, it shows that a rubble pile body can never achieve an aspect ratio beyond 21:1, at least when it consists of equal-sized, spherical components".

Under the section "4.2 Necessary conditions", in paragraph 2, the first line should read as "This condition is in direct competition for the existence condition for the Euler Resting configuration, summarized in Eq. 28. Comparing Eq. 46 with Eq. 28, one finds that the angular momentum for the necessary condition to be satisfied exceeds the existence condition limit for $N \geq 31$ ".

Under the section "4.3 Sufficient conditions", in paragraph 1, the last line should read as "Carrying out the computation shows that for $N=5$ the sufficiency condition is definitely satisfied when the system is evaluated at the fission condition; however, at $N=22$ and higher, it is violated with at least one negative eigenvalue".

Under the section "4.3 Sufficient conditions", the last paragraph should read as "Note that the case $N=2$ is trivially stable when it exists and that the cases $N=3$, 4 have been studied in detail previously. In the following we investigate in detail the case of $N=5$ ".

Under the section "5 Discussion", the last paragraph should read as "That the Euler Resting configuration is only stable up to $N=21$ places a limit on the elongation that a collection of resting particles can have at 21:1. A fundamental question of interest for future studies is whether a similar elongation limit holds when systems of more bodies are considered, arranged to have additional components stacked on each other in directions transverse to the long axis". 